\title{
Robust International Portfolio Optimization with Worst-Case Mean-LPM
}

\author{
Fei Luan (D), Weiguo Zhang (D), and Yongjun Liu $(\mathbb{C}$ \\ School of Business Administration, South China University of Technology, Guangzhou 510641, China \\ Correspondence should be addressed to Weiguo Zhang; wgzhang@scut.edu.cn
}

Received 8 October 2021; Accepted 12 January 2022; Published 23 February 2022

Academic Editor: Guoqiang Wang

Copyright (c) 2022 Fei Luan et al. This is an open access article distributed under the Creative Commons Attribution License, which permits unrestricted use, distribution, and reproduction in any medium, provided the original work is properly cited.

\begin{abstract}
This paper proposes a robust international portfolio optimization model with the consideration of worst-case lower partial moment (LPM) and worst-case mean return. In our model, we assume that the distributions and the first- and second-order moments of distributions of returns of assets and exchange rates are all ambiguous. The proposed model can be reformulated into an equivalent semidefinite programming (SDP) problem, which is computationally tractable. For investigation of the performance of our model, we also give two benchmark models. The first benchmark model is a scenario-based model which uses historical observations of returns to approximate the future distributions. The second benchmark model only considers the ambiguity of distributions but does not consider the ambiguity of the first- and second-order moments of distributions. We conduct empirical experiments in a rolling forward way to evaluate the out-of-sample performances of our proposed model, the two benchmark models, and an equally weighted model using the return measures and various risk-adjusted return measures. The result shows that our model has the best performance. It verifies that investors can obtain benefits when employing the robust model and considering the ambiguity of the first- and second-order moments of distributions.
\end{abstract}

\section{Introduction}

In order to capture the diversification benefits of international financial markets, institutional and individual investors tend to invest part of their money in the financial markets of other countries or regions using different currencies. The correlations of returns of assets in other countries or regions are often lower than those in just one country, so international asset allocation may reduce risk [1-5]. Generally, the distribution of international portfolio return is asymmetry. It is well known that variance is not an appropriate measure to evaluate the risk of asymmetry distributions, whereas downside risk measures can measure the risk of asymmetry distributions effectively. Among various downside risk measures, lower partial moments (LPM) are comprehensive and sensible [6]. The definition of LPM is introduced by Bawa [7], Bawa and Lindenberg [8], and Fishburn [9]. In order to compute LPM, we need to know the distributions of future security returns beforehand. However, for investors, the distributions of future security returns are usually unknown or cannot be estimated accurately. Even if they acquire the actual distributions of future security returns, the computation of LPM is also a difficult task. To deal with these problems, some researchers employ robust optimization techniques to portfolio selection models using LPM as the risk measure [10-14]. Their researches focus on the portfolio selection problems in one country and do not consider the international portfolio selection problems with the risk of exchange rates. Meanwhile, their researches only consider the worst-case LPM and do not consider the worst-case mean return. Intuitively, the worst-case mean return can also give investors helpful instructions for making investment decisions.

In this paper, we build a robust international portfolio optimization model with worst-case LPM as the risk measure and consider the worst-case mean return. We assume that the distributions and the first- and second-order moments of distributions of future returns of assets and exchange rates are all ambiguous. Using robust optimization techniques, we reformulate our model into an equivalent 
semidefinite programming (SDP) problem. In order to evaluate the performance of our model, we also give two benchmark models. In the first benchmark model, we use historical return observations to form empirical distributions of future returns and build an international portfolio optimization model based on these empirical distributions. In the second benchmark model, we assume that the distributions of future returns are ambiguous, but the first- and second-order moments are known. Then, we conduct empirical experiments using the return measures and various risk-adjusted return measures to assess the performances of our model, the two benchmark models, and the equally weighted model.

This paper is organized as follows. In Section 2, we propose the robust international portfolio optimization model with worst-case LPM and mean return under a distributional ambiguity set where the distributions and the first- and second-order moments are ambiguous. We derive an equivalent SDP reformulation of this model. In Section 3, we present the two benchmark models. In Section 4, we conduct empirical experiments to evaluate the performance of our model in comparison with the two benchmark models and the equally weighted model using the return measures and various risk-adjusted return measures. Section 5 gives the conclusions of this paper.

1.1. Notation. In this paper, vectors are denoted by lowercase boldface letters, and matrices are denoted by uppercase boldface letters. We use $\mathbb{R}^{n}$ to denote the space of vectors of real numbers with dimension $n$ and $\mathbb{S}^{n}$ to denote the space of symmetric matrices with dimension $n$. For any two matrices $\mathbf{X}, \mathbf{Y} \in \mathbb{S}^{n}$, we use $\langle\mathbf{X}, \mathbf{Y}\rangle=\operatorname{trace}(X Y)$ to denote the trace scalar product, and the relation $\mathbf{X} \geqslant \mathbf{Y}$ represents that $\mathbf{X}-\mathbf{Y}$ is positive semidefinite. Random variables are denoted by symbols with tildes, whereas the realizations of them are denoted by symbols without tildes.

\section{Model Formulation}

In the international financial markets, we assume that an investor plans to invest in the stock markets of $n$ foreign countries or overseas regions where people use different currencies to the investor's domestic currency. We denote that the return of the asset in the $i$-th country or region is $\widetilde{s}_{i}$, and the return of the exchange rate of the $i$-th country or region is $\widetilde{c}_{i}$, where $i=1,2, \ldots, n$. Then, the return of the $i$-th asset in the investor's domestic currency can be obtained as

$$
\left(1+\widetilde{s}_{i}\right)\left(1+\widetilde{c}_{i}\right)-1=\widetilde{s}_{i}+\widetilde{c}_{i}+\widetilde{s}_{i} \widetilde{c}_{i} .
$$

We assume that the weight of money in the domestic currency of the investor invested in the $i$-th asset is $w_{i}$, and the sum of $w_{i}, i=1,2, \ldots, n$, equals 1 . Then, the total return of the international portfolio $\mathbf{w}=\left(w_{1}, w_{2}, \ldots, w_{n}\right)^{T}$ can be written as

$$
\sum_{i=1}^{n}\left(\widetilde{s}_{i}+\widetilde{c}_{i}+\widetilde{s}_{i} \widetilde{c}_{i}\right) w_{i}
$$

For convenience, we denote that

$$
\widetilde{\xi}=\left(\widetilde{s}_{1}, \widetilde{s}_{2}, \ldots, \widetilde{s}_{n}, \widetilde{c}_{1}, \widetilde{c}_{2}, \ldots, \widetilde{c}_{n}\right)^{T},
$$

which combines the returns of assets and exchange rates in one vector. We also denote that

$$
\begin{aligned}
& \mathbf{W}=\left[\begin{array}{llll}
w_{1} & & & \\
& w_{2} & & \\
& & \ddots & \\
& & & \\
& & & w_{n}
\end{array}\right], \\
& \mathbf{H}=\left[\begin{array}{ll}
0_{n \times n} & \frac{1}{2} \mathbf{W} \\
\frac{1}{2} \mathbf{W} & 0_{n \times n}
\end{array}\right], \\
& \mathbf{B}_{1}=\left[\begin{array}{c}
\mathbf{I}_{n \times n} \\
0_{n \times n}
\end{array}\right] \text {, } \\
& \mathbf{B}_{2}=\left[\begin{array}{l}
0_{n \times n} \\
\mathbf{I}_{n \times n}
\end{array}\right] \text {. }
\end{aligned}
$$

Then, (2) can be rewritten as

$$
r(\mathbf{w}, \widetilde{\boldsymbol{\xi}})=\widetilde{\boldsymbol{\xi}}^{T} \mathbf{B}_{1} \mathbf{w}+\widetilde{\boldsymbol{\xi}}^{T} \mathbf{B}_{2} \mathbf{w}+\widetilde{\boldsymbol{\xi}}^{T} \mathbf{H} \widetilde{\boldsymbol{\xi}} .
$$

We employ first-order lower partial moment to measure the risk of international portfolios. For a given benchmark return $a$, the first-order LPM can be written as follows:

$$
\operatorname{LPM}(\mathbf{w}, P)=E_{P}\left[(a-r(\mathbf{w}, \widetilde{\xi}))_{+}\right],
$$

where $P$ is the distribution of $\widetilde{\xi}$. In practice, investors usually cannot know $P$ accurately beforehand. Thus, some researchers use historical observations of returns to form an empirical approximation of $P$. We assume that investors can obtain $m$ historical observations, which are denoted by $\widehat{\xi}_{1}, \widehat{\xi}_{2}$, $\ldots, \widehat{\xi}_{m}$. The empirical approximation $\widehat{P}$ of $P$ is typically formed as follows:

$$
\widehat{P}\left(\widetilde{\xi}=\widehat{\xi}_{i}\right)=\frac{1}{m}, \quad i=1,2, \ldots, m .
$$

Under $\widehat{P}$, the LPM in (6) can be rewritten as

$$
\operatorname{LPM}(\mathbf{w}, \widehat{P})=\sum_{i=1}^{m} \frac{1}{m}\left[\left(a-r\left(\mathbf{w}, \widehat{\xi}_{i}\right)\right)_{+}\right] .
$$

If $m$ is relatively large, according to the law of large numbers, the gap between (6) and its scenario-based version (8) can be small. But the number of observations that investors can acquire is usually small and cannot satisfy the requirement of the law of large numbers. Instead, some researchers use historical observations of returns to form a distributional ambiguity set of future returns. A popular ambiguity set that considers the ambiguity of the 
distributions and the first- and second-order moments of distributions is proposed by Delage and Ye [15]. This ambiguity set denoted by $\mathscr{P}_{1}$ can be described as follows:

$$
\mathscr{P}_{1}\left(\widehat{\boldsymbol{\mu}}, \widehat{\boldsymbol{\Sigma}}, \lambda_{1}, \lambda_{2}\right)=\left\{\begin{array}{c}
\mathbb{P}\left(\widetilde{\xi} \in \mathbb{R}^{2 n}\right)=1 \\
P \in \mathscr{M} \mid(\mathbb{E}(\widetilde{\boldsymbol{\xi}})-\widehat{\mu})^{T} \widehat{\boldsymbol{\Sigma}}^{-1}(\mathbb{E}(\widetilde{\boldsymbol{\xi}})-\widehat{\boldsymbol{\mu}}) \leq \lambda_{1} \\
\mathbb{E}\left[(\widetilde{\boldsymbol{\xi}}-\widehat{\boldsymbol{\mu}})(\widetilde{\boldsymbol{\xi}}-\widehat{\boldsymbol{\mu}})^{T}\right] \leqq \lambda_{2} \widehat{\boldsymbol{\Sigma}}
\end{array}\right\},
$$

where $\mathscr{M}$ is the set of all probability measures on the measurable space $\left(\mathbb{R}^{2 n}, \mathscr{B}\right)$, with $\mathscr{B}$ being the Borel $\sigma$-algebra on $\mathbb{R}^{2 n}, \widehat{\mu}$ is the sample-based mean return, $\widehat{\Sigma}$ is the sample-based covariance matrix, $\lambda_{1}$ reflects the ambiguity size of mean return, and $\lambda_{2}$ reflects the ambiguity size of the covariance matrix. The worst-case LPM with respect to the ambiguity set $\mathscr{P}_{1}$ denoted by $W \operatorname{LPM}\left(\mathbf{w}, \mathscr{P}_{1}\right)$ can be defined as

$$
\operatorname{WLPM}\left(\mathbf{w}, \mathscr{P}_{1}\right)=\max _{P \in \mathscr{P}_{1}} \operatorname{LPM}\left(\mathbf{w}, \mathscr{P}_{1}\right)
$$

Since the mean return of the international portfolio can also give investors useful instructions for decision-making, we add the worst-case mean return in the objective function of our model. We give the definition of worst-case mean return with respect to $\mathscr{P}_{1}$ denoted by $W \operatorname{Return}\left(\mathbf{w}, \mathscr{P}_{1}\right)$ as follows:

$$
W \operatorname{Return}\left(\mathbf{w}, \mathscr{P}_{1}\right)=\min _{P \in \mathscr{P}_{1}} E_{P}[r(\mathbf{w}, \widetilde{\xi})] .
$$

The robust international portfolio optimization model using worst-case LPM as the risk measure and considering worst-case mean return under $\mathscr{P}_{1}$ can be built as follows:

$$
(\mathrm{RIML}) \min _{\mathbf{w}} \lambda \cdot \mathrm{WLPM}\left(\mathbf{w}, \mathscr{P}_{1}\right)-(1-\lambda) \cdot W \operatorname{Return}\left(\mathbf{w}, \mathscr{P}_{1}\right) .
$$

$$
\text { s.t. } \mathbf{w} \in \mathbb{R}^{n}, \mathbf{w}^{T} \mathbf{e}=1, \mathbf{w} \geq 0,
$$

where $\lambda$ is the risk aversion coefficient of investors and $\mathbf{e}$ denotes the vector of $1 \mathrm{~s}$ with dimension $n$. Problem (12) cannot be solved directly; thus, we need to derive its equivalent reformulation, which is computationally tractable. In the following, we first give the equivalent SDP reformulation of $W L P M\left(\mathbf{w}, \mathscr{P}_{1}\right)$ defined by (10); then, we give the equivalent SDP reformulation of $W \operatorname{Return}\left(\mathbf{w}, \mathscr{P}_{1}\right)$ defined by (11).

Theorem 1. WLPM $\left(\mathbf{w}, \mathscr{P}_{1}\right)$ defined by (10) is equal to the optimal objective function value of the following SDP problem:

$$
\begin{aligned}
& \min _{\left\{p_{1}, \mathbf{U}, \mathbf{V}, \mathbf{v}, s\right\}} p_{1}+\lambda_{2}\langle\mathbf{U}, \widehat{\Sigma}\rangle \\
& \text { s.t. } \mathbf{U} \geqslant 0 \text {, } \\
& {\left[\begin{array}{cc}
\mathbf{V} & \mathbf{v} \\
\mathbf{v}^{T} & \boldsymbol{s}
\end{array}\right] \geqslant 0} \\
& {\left[\begin{array}{cc}
\mathbf{U} & \frac{1}{2}(-2 \mathbf{U} \hat{\boldsymbol{\mu}}-2 \mathbf{v}) \\
\frac{1}{2}(-2 \mathbf{U} \widehat{\boldsymbol{\mu}}-2 \mathbf{v})^{T} & p_{1}+\widehat{\boldsymbol{\mu}}^{T} \mathbf{U} \widehat{\boldsymbol{\mu}}-\langle\mathbf{V}, \widehat{\boldsymbol{\Sigma}}\rangle+2 \mathbf{v}^{T} \widehat{\boldsymbol{\mu}}-\lambda_{1} s
\end{array}\right] \geqslant 0,} \\
& {\left[\begin{array}{cc}
\mathbf{U}+\mathbf{H} & \frac{1}{2}\left(-2 \mathbf{U} \widehat{\boldsymbol{\mu}}-2 \mathbf{v}+\mathbf{B}_{1} \mathbf{w}+\mathbf{B}_{2} \mathbf{w}\right) \\
\frac{1}{2}\left(-2 \mathbf{U} \widehat{\boldsymbol{\mu}}-2 \mathbf{v}+\mathbf{B}_{1} \mathbf{w}+\mathbf{B}_{2} \mathbf{w}\right)^{T} & p_{1}+\widehat{\boldsymbol{\mu}}^{T} \mathbf{U} \widehat{\boldsymbol{\mu}}-\langle\mathbf{V}, \widehat{\boldsymbol{\Sigma}}\rangle+2 \mathbf{v}^{T} \widehat{\boldsymbol{\mu}}-\lambda_{1} s-a
\end{array}\right] \geqslant 0 .} \\
& \min _{P}-\int_{\mathbb{R}^{2 n}}(a-r(\mathbf{w}, \widetilde{\xi}))_{+} d P(\widetilde{\boldsymbol{\xi}}), \\
& \text { s.t. } \int_{\mathbb{R}^{2 n}} \mathrm{dP}(\tilde{\xi})=1 \text {, } \\
& \int_{\mathbb{R}^{2 n}}(\widetilde{\boldsymbol{\xi}}-\widehat{\boldsymbol{\mu}})(\widetilde{\boldsymbol{\xi}}-\widehat{\boldsymbol{\mu}})^{T} d P(\widetilde{\boldsymbol{\xi}}) \prec \lambda_{2} \widehat{\boldsymbol{\Sigma}}, \\
& \int_{\mathbb{R}^{2 n}}\left[\begin{array}{cc}
\widehat{\Sigma} & (\widetilde{\boldsymbol{\xi}}-\widehat{\boldsymbol{\mu}}) \\
(\widetilde{\boldsymbol{\xi}}-\widehat{\boldsymbol{\mu}})^{T} & \lambda_{1}
\end{array}\right] d P(\widetilde{\boldsymbol{\xi}}) \geqslant 0, \\
& \min _{P \in \mathscr{P}_{1}}-E_{P}\left[(a-r(\mathbf{w}, \widetilde{\boldsymbol{\xi}}))_{+}\right],
\end{aligned}
$$

which can be written as

Then, we shall derive the equivalent SDP reformulation of the following: 


$$
P \in \mathscr{M} \text {. }
$$

We denote the dual variable of constraint (16b) by $p_{1}$, that of constraint (16c) by $\mathbf{U}$, and that of constraint (16d) by

$$
\left[\begin{array}{cc}
\mathbf{V} & v \\
\mathbf{v}^{T} & s
\end{array}\right],
$$

where $p_{1} \in \mathbb{R} ; \mathbf{U} \in \mathbb{S}^{2 n} ; \mathbf{U} \geqslant 0 ; \mathbf{V} \in \mathbb{S}^{2 n} ; \mathbf{v} \in \mathbb{R}^{2 n} ; s \in \mathbb{R}$;

$$
\left[\begin{array}{cc}
\mathbf{V} & v \\
\mathbf{v}^{T} & s
\end{array}\right] \geqslant 0 .
$$

The dual reformulation of problem (16a) can be written as follows:

$$
\begin{gathered}
\max _{\left\{p_{1}, \mathbf{U}, \mathbf{V}, \mathbf{v}, s\right\}}-p_{1}-\lambda_{2}\langle\mathbf{U}, \widehat{\Sigma}\rangle, \\
\text { s.t. } p_{1}+(\widetilde{\boldsymbol{\xi}}-\widehat{\boldsymbol{\mu}})^{T} \mathbf{U}(\widetilde{\boldsymbol{\xi}}-\widehat{\boldsymbol{\mu}})-\langle\mathbf{V}, \widehat{\Sigma}\rangle-2 \mathbf{v}^{T}(\widetilde{\boldsymbol{\xi}}-\widehat{\boldsymbol{\mu}}) \\
-\lambda_{1} \mathrm{~s} \geq(a-r(\mathbf{w}, \widetilde{\boldsymbol{\xi}}))_{+}, \quad \forall \widetilde{\boldsymbol{\xi}} \in \mathbb{R}^{2 \mathrm{n}} .
\end{gathered}
$$

The Dirac measure $\delta_{\widehat{\mu}}$ is the measure of mass one at the point $\widehat{\mu}$. Obviously, $\delta_{\widehat{\mu}}$ lies in the relative interior of the feasible set of problem (16). According to the weak version of Proposition 3.4 in Shapiro [16], we can deduce that there is no dual gap between problems (16) and (19). Thus, the optimal objective function value of problem (16) is equal to that of problem (19). Constraint (19b) is equivalent to the following two constraints:

$$
\begin{aligned}
p_{1} & +(\widetilde{\boldsymbol{\xi}}-\widehat{\boldsymbol{\mu}})^{T} \mathbf{U}(\widetilde{\boldsymbol{\xi}}-\widehat{\boldsymbol{\mu}})-\langle\mathbf{V}, \widehat{\Sigma}\rangle-2 \mathbf{v}^{T}(\widetilde{\boldsymbol{\xi}}-\widehat{\boldsymbol{\mu}}) \\
& -\lambda_{1} s \geq 0, \quad \forall \widetilde{\boldsymbol{\xi}} \in \mathbb{R}^{2 n} . \\
p_{1} & +(\widetilde{\boldsymbol{\xi}}-\widehat{\boldsymbol{\mu}})^{T} \mathbf{U}(\widetilde{\boldsymbol{\xi}}-\widehat{\boldsymbol{\mu}})-\langle\mathbf{V}, \widehat{\Sigma}\rangle-2 \mathbf{v}^{T}(\widetilde{\boldsymbol{\xi}}-\widehat{\boldsymbol{\mu}}) \\
& -\lambda_{1} s \geq a-r(\mathbf{w}, \widetilde{\boldsymbol{\xi}}), \quad \forall \widetilde{\xi} \in \mathbb{R}^{2 n} .
\end{aligned}
$$

Equation (20) is equivalent to the matrix inequality (13d), and (21) is equivalent to the matrix inequality (13e). Thus, we complete the proof of this theorem.

Theorem 2. WReturn $\left(\mathbf{w}, \mathscr{P}_{1}\right)$ defined in (11) is equal to the optimal objective function value of the following SDP problem:

$$
\begin{aligned}
& \max _{\left\{q_{1}, \mathbf{Q}, \mathbf{M}, \mathbf{h}, z\right\}}-q_{1}-\lambda_{2}\langle\mathbf{Q}, \widehat{\Sigma}\rangle, \\
& \text { s.t. } \mathbf{Q} \geqslant 0 \text {, } \\
& {\left[\begin{array}{ll}
\mathbf{M} & \mathbf{h} \\
\mathbf{h}^{T} & z
\end{array}\right] \geqslant 0,} \\
& {\left[\begin{array}{cc}
\mathbf{H}+\mathbf{Q} & \frac{1}{2}\left(\mathbf{B}_{1} \mathbf{w}+\mathbf{B}_{2} \mathbf{w}-2 \mathbf{Q} \widehat{\boldsymbol{\mu}}-2 \mathbf{h}\right) \\
\frac{1}{2}\left(\mathbf{B}_{1} \mathbf{w}+\mathbf{B}_{2} \mathbf{w}-2 \mathbf{Q} \widehat{\boldsymbol{\mu}}-2 \mathbf{h}\right)^{T} & q_{1}+\widehat{\boldsymbol{\mu}}^{T} \mathbf{Q} \widehat{\boldsymbol{\mu}}-\langle\mathbf{M}, \widehat{\boldsymbol{\Sigma}}\rangle+2 \mathbf{h}^{T} \widehat{\boldsymbol{\mu}}-\lambda_{1} z
\end{array}\right] \geqslant 0 .}
\end{aligned}
$$

Proof: . WReturn $\left(\mathbf{w}, \mathscr{P}_{1}\right)$ defined by (11) can be written in the following formulation:

$$
\begin{aligned}
& \min _{P} \int_{\mathbb{R}^{2 n}}\left(\widetilde{\boldsymbol{\xi}}^{T} \mathbf{B}_{1} \mathbf{w}+\widetilde{\boldsymbol{\xi}}^{T} \mathbf{B}_{2} \mathbf{w}+\widetilde{\boldsymbol{\xi}}^{T} \mathbf{H} \tilde{\boldsymbol{\xi}}\right) d P(\widetilde{\xi}) \\
& \text { s.t. } \int_{\mathbb{R}^{2 n}} \mathrm{~d} P(\widetilde{\boldsymbol{\xi}})=1 \\
& \int_{\mathbb{R}^{2 n}}(\widetilde{\boldsymbol{\xi}}-\widehat{\boldsymbol{\mu}})(\widetilde{\boldsymbol{\xi}}-\widehat{\mu})^{T} d P(\widetilde{\boldsymbol{\xi}}) \prec \lambda_{2} \widehat{\boldsymbol{\Sigma}} \\
& \int_{\mathbb{R}^{2 n}}\left[\begin{array}{c}
\widehat{\boldsymbol{\Sigma}} \quad(\widetilde{\boldsymbol{\xi}}-\widehat{\boldsymbol{\mu}}) \\
(\widetilde{\boldsymbol{\xi}}-\widehat{\boldsymbol{\mu}})^{T} \\
\lambda_{1}
\end{array}\right] d P(\widetilde{\boldsymbol{\xi}}) \geqslant 0 \\
& P \in \mathscr{M} .
\end{aligned}
$$

Similar to the proof of Theorem 1, we denote the dual variable of constraint (23b) by $q_{1}$, that of constraint (16c) by $Q$, and that of constraint (16d) by

$$
\left[\begin{array}{ll}
\mathbf{M} & \mathbf{h} \\
\mathbf{h}^{T} & z
\end{array}\right],
$$

where $q_{1} \in \mathbb{R} ; \mathbf{Q} \in \mathbb{S}^{2 n} ; \mathbf{Q} \geqslant 0 ; \mathbf{M} \in \mathbb{S}^{2 n} ; \mathbf{h} \in \mathbb{R}^{2 n} ; z \in \mathbb{R}$;

$$
\left[\begin{array}{ll}
\mathbf{M} & \mathbf{h} \\
\mathbf{h}^{T} & z
\end{array}\right] \geqslant 0 .
$$

The dual reformulation of problem (16) can be written as

$$
\begin{gathered}
\max _{\left\{q_{1}, \mathbf{Q}, \mathbf{M}, \mathbf{h}, z\right\}}-q_{1}-\lambda_{2}\langle\mathbf{Q}, \widehat{\Sigma}\rangle, \\
\text { s.t. } \quad \mathbf{Q} \geqslant 0, \\
{\left[\begin{array}{ll}
\mathbf{M} & \mathbf{h} \\
\mathbf{h}^{T} & z
\end{array}\right] \geqslant 0,}
\end{gathered}
$$

$$
\begin{array}{r}
\widetilde{\boldsymbol{\xi}}^{T} \mathbf{B}_{1} \mathbf{w}+\widetilde{\boldsymbol{\xi}}^{T} \mathbf{B}_{2} \mathbf{w}+\widetilde{\boldsymbol{\xi}}^{T} \mathbf{H} \widetilde{\boldsymbol{\xi}}+q_{1}+(\widetilde{\boldsymbol{\xi}}-\widehat{\boldsymbol{\mu}})^{T} \mathbf{Q}(\widetilde{\boldsymbol{\xi}}-\widehat{\boldsymbol{\mu}}) \\
-\langle\mathbf{M}, \widehat{\Sigma}\rangle-2 \mathbf{h}^{T}(\widetilde{\boldsymbol{\xi}}-\widehat{\boldsymbol{\mu}})-\lambda_{1} z \geq 0, \quad \forall \widetilde{\xi} \in \mathbb{R}^{2 n} .
\end{array}
$$


Similar to the proof of Theorem 1, there is no dual gap between problems (23) and (26); thus, the optimal objective function values of the two problems are the same. The equivalent matrix inequality of constraint (26d) is (22d); thus, we complete the proof of this theorem.

With Theorems 1 and 2, we can easily obtain the equivalent SDP reformulation of problem RIML (12), and the final formulation is as follows:

$$
\begin{aligned}
& \min _{\left\{\begin{array}{l}
p_{1}, \mathbf{U}, \mathbf{V}, \mathbf{v}, s, \\
q_{1}, \mathbf{Q}, \mathbf{M}, \mathbf{h}, z
\end{array}\right\}} \lambda \cdot\left(p_{1}+\lambda_{2}\langle\mathbf{U}, \widehat{\Sigma}\rangle\right)+(1-\lambda) \cdot\left(q_{1}+\lambda_{2}\langle\mathbf{Q}, \widehat{\Sigma}\rangle\right), \\
& \text { s.t. (12b), (13b), (13c), (13d), (13e), (22b), (22c), (22d). }
\end{aligned}
$$

\section{Two Benchmark Models}

In order to assess the performance of our model RIML, we present two benchmark models in this section. The first benchmark model denoted by SIML is based on empirical distributions approximated by historical samples of returns. The approximated distribution $\widehat{P}$ is described in (7), and LPM under $\widehat{P}$ is shown in (8). The return of the international portfolio under $\widehat{P}$ can be written as

$$
\operatorname{Return}(\mathbf{w}, \widehat{P})=\frac{1}{m} \sum_{i=1}^{m} r\left(\mathbf{w}, \widehat{\xi}_{i}\right) .
$$

The scenario-based international portfolio optimization model with mean-LPM denoted by SIML is built as

$(\operatorname{SIML}) \min _{\mathbf{w}} \lambda \cdot \operatorname{LPM}(\mathbf{w}, \widehat{\mathrm{P}})-(1-\lambda) \cdot \operatorname{Return}(\mathbf{w}, \widehat{\mathrm{P}})$,

$$
\text { s.t. } \mathbf{w} \in \mathbb{R}^{\mathrm{n}}, \mathbf{e}^{\mathrm{T}} \mathbf{w}=1, \mathbf{w} \geq 0,
$$

where $\operatorname{LPM}(\mathbf{w}, \widehat{P})$ is defined by $(8)$ and $\operatorname{Return}(\mathbf{w}, \widehat{P})$ is defined by (28). In the second benchmark model denoted by $\mathrm{RIML}^{-}$, we assume that the distributions of returns of assets and exchange rates are ambiguous, but the first- and secondorder moments of distributions are determined beforehand. The corresponding ambiguity set $\mathscr{P}_{2}$ is described as follows:

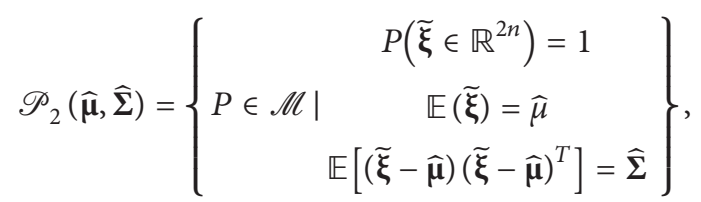

where the definitions of $\mathscr{M}$, $\widehat{\boldsymbol{\mu}}$, and $\widehat{\boldsymbol{\Sigma}}$ are the same as those of (9). Under $\mathscr{P}_{2}$, RIML ${ }^{-}$can be written as

$\left(\operatorname{RIML}^{-}\right) \min _{\mathbf{w}} \lambda \cdot W L P M\left(\mathbf{w}, \mathscr{P}_{2}\right)-(1-\lambda) \cdot W \operatorname{Return}\left(\mathbf{w}, \mathscr{P}_{2}\right)$,

$$
\text { s.t. } \mathbf{w} \in \mathbb{R}^{n}, \mathbf{w}^{T} \mathbf{e}=1, \mathbf{w} \geq 0 \text {, }
$$

where

$$
\begin{aligned}
& \operatorname{WLPM}\left(\mathbf{w}, \mathscr{P}_{2}\right)=\max _{P \in \mathscr{P}_{2}} \operatorname{LPM}(\mathbf{w}, P), \\
& W \operatorname{Return}\left(\mathbf{w}, \mathscr{P}_{2}\right)=\min _{P \in \mathscr{P}_{2}} E_{P}(r(\mathbf{w}, \widetilde{\xi})) .
\end{aligned}
$$

In the following, we also try to derive the equivalent SDP reformulations of $\operatorname{WLPM}\left(\mathbf{w}, \mathscr{P}_{2}\right)$ defined by (32) and $W \operatorname{Return}\left(\mathbf{w}, \mathscr{P}_{2}\right)$ defined by (33).

Theorem 3. WLPM $\left(\mathbf{w}, \mathscr{P}_{2}\right)$ defined by (32) is equal to the optimal objective function value of the following SDP problem:

$$
\begin{aligned}
& \min _{\left\{p_{1}, \mathbf{v}, \mathbf{U}\right\}} p_{1}+\mathbf{v}^{T} \widehat{\mu}+\langle\mathbf{U}, \widehat{\Sigma}\rangle, \\
& \text { s.t. }\left[\begin{array}{cc}
\mathbf{U}+\mathbf{H} & \frac{1}{2}\left(\mathbf{v}-2 \mathbf{U} \hat{\boldsymbol{\mu}}+\mathbf{B}_{1} \mathbf{w}+\mathbf{B}_{2} \mathbf{w}\right) \\
\frac{1}{2}\left(\mathbf{v}-2 \mathbf{U} \widehat{\boldsymbol{\mu}}+\mathbf{B}_{1} \mathbf{w}+\mathbf{B}_{2} \mathbf{w}\right)^{\mathrm{T}} & p_{1}+\widehat{\boldsymbol{\mu}}^{\mathrm{T}} \mathbf{U} \hat{\boldsymbol{\mu}}-a
\end{array}\right] \geqslant 0,
\end{aligned}
$$

$$
\left[\begin{array}{cc}
\mathbf{U} & \frac{1}{2}(\mathbf{v}-2 \mathbf{U} \widehat{\boldsymbol{\mu}}) \\
\frac{1}{2}(\mathbf{v}-2 \mathbf{U} \widehat{\boldsymbol{\mu}})^{T} & p_{1}+\widehat{\boldsymbol{\mu}}^{T} \mathbf{U} \widehat{\boldsymbol{\mu}}
\end{array}\right] \succcurlyeq 0 .
$$

Proof. According to (32), WLPM (w, $\left.\mathscr{P}_{2}\right)$ can be rewritten as

$$
-\min _{P \in \mathscr{P}_{2}}-E_{P}\left[(a-r(\mathbf{w}, \widetilde{\xi}))_{+}\right] .
$$

Thus, we first study the equivalent SDP reformulation of the following problem:

$$
\min _{P \in \mathscr{P}_{2}}-E_{P}\left[(a-r(\mathbf{w}, \tilde{\xi}))_{+}\right],
$$

which can be rewritten as

$$
\begin{gathered}
\min _{P}-\int_{\mathbb{R}^{2 n}}\left[(a-r(\mathbf{w}, \widetilde{\boldsymbol{\xi}}))_{+}\right] d P(\widetilde{\boldsymbol{\xi}}), \\
\text { s.t. } \int_{\mathbb{R}^{2 n}} d P(\widetilde{\boldsymbol{\xi}})=1, \\
\int_{\mathbb{R}^{2 n}} \tilde{\boldsymbol{\xi}} d P(\tilde{\boldsymbol{\xi}})=\widehat{\boldsymbol{\mu}}, \\
\int_{\mathbb{R}^{2 n}}(\widetilde{\boldsymbol{\xi}}-\widehat{\boldsymbol{\mu}})(\widetilde{\boldsymbol{\xi}}-\widehat{\boldsymbol{\mu}})^{T} d P(\widetilde{\boldsymbol{\xi}})=\widehat{\Sigma} .
\end{gathered}
$$

We set the dual variable of constraint (37b) as $p_{1}$, that of constraint (37c) as $\mathbf{v}$, and that of constraint (37d) as $\mathbf{U}$. The dual reformulation of problem (37) can be written as follows: $\max _{\left\{p_{1}, \mathbf{v}, \mathbf{U}\right\}}-p_{1}-\mathbf{v}^{T} \widehat{\mu}-\langle\mathbf{U}, \widehat{\Sigma}\rangle$,

s.t. $p_{1}+\mathbf{v}^{\mathrm{T}} \widetilde{\boldsymbol{\xi}}+(\widetilde{\boldsymbol{\xi}}-\widehat{\boldsymbol{\mu}})^{\mathrm{T}} \mathbf{U}(\widetilde{\boldsymbol{\xi}}-\widehat{\boldsymbol{\mu}}) \geq(a-r(\mathbf{w}, \widetilde{\boldsymbol{\xi}}))_{+}, \quad \forall \widetilde{\boldsymbol{\xi}} \in \mathbb{R}^{2 n}$.

Obviously, there is no dual gap between problems (37) and (38). Thus, the two problems (37) and (38) have the same optimal objective function value. We note that constraint (38b) is equivalent to the following two inequalities: 


$$
\begin{aligned}
& p_{1}+\mathbf{v}^{T} \widetilde{\boldsymbol{\xi}}+(\widetilde{\boldsymbol{\xi}}-\widehat{\boldsymbol{\mu}})^{T} \mathbf{U}(\widetilde{\boldsymbol{\xi}}-\widehat{\boldsymbol{\mu}}) \geq a-r(\mathbf{w}, \widetilde{\boldsymbol{\xi}}), \quad \forall \widetilde{\xi} \in \mathbb{R}^{2 n}, \\
& p_{1}+\mathbf{v}^{T} \widetilde{\boldsymbol{\xi}}+(\widetilde{\boldsymbol{\xi}}-\widehat{\boldsymbol{\mu}})^{T} \mathbf{U}(\widetilde{\boldsymbol{\xi}}-\widehat{\boldsymbol{\mu}}) \geq 0, \quad \forall \widetilde{\xi} \in \mathbb{R}^{2 n} .
\end{aligned}
$$

The equivalent matrix inequality of (39) is (34b), and that of (40) is (34c). Thus, we complete the proof of this theorem.

Theorem 4. WReturn (w, $\left.\mathscr{P}_{2}\right)$ defined by (33) is equal to the optimal objective function value of the following SDP problem:

$$
\begin{aligned}
& \max _{\left\{q_{1}, \mathbf{h}, \mathbf{Q}\right\}}-q_{1}-\mathbf{h}^{T} \widehat{\mu}-\langle\mathbf{Q}, \widehat{\Sigma}\rangle, \\
& \text { s.t. }\left[\begin{array}{cc}
\mathbf{H}+\mathbf{Q} & \frac{1}{2}\left(\mathbf{B}_{1} \mathbf{w}+\mathbf{B}_{2} \mathbf{w}+\mathbf{h}-2 \mathbf{Q} \widehat{\boldsymbol{\mu}}\right) \\
\frac{1}{2}\left(\mathbf{B}_{1} \mathbf{w}+\mathbf{B}_{2} \mathbf{w}+\mathbf{h}-2 \mathbf{Q} \widehat{\boldsymbol{\mu}}\right)^{T} & q_{1}+\widehat{\boldsymbol{\mu}}^{T} \mathbf{Q} \widehat{\boldsymbol{\mu}}
\end{array}\right] \geqslant 0 .
\end{aligned}
$$

Proof: $W \operatorname{Return}\left(\mathbf{w}, \mathscr{P}_{2}\right)$ defined by (33) can be rewritten as follows:

$$
\begin{gathered}
\min _{P} \int_{\mathbb{R}^{2 n}}\left(\widetilde{\boldsymbol{\xi}}^{T} \mathbf{B}_{1} \mathbf{w}+\widetilde{\boldsymbol{\xi}}^{T} \mathbf{B}_{2} \mathbf{w}+\widetilde{\boldsymbol{\xi}}^{T} \mathbf{H} \tilde{\boldsymbol{\xi}}\right) d P(\widetilde{\boldsymbol{\xi}}), \\
\text { s.t. } \int_{\mathbb{R}^{2 n}} d P(\widetilde{\boldsymbol{\xi}})=1, \\
\int_{\mathbb{R}^{2 n}} \tilde{\boldsymbol{\xi}} d P(\widetilde{\boldsymbol{\xi}})=\widehat{\boldsymbol{\mu}}, \\
\int_{\mathbb{R}^{2 n}}(\widetilde{\boldsymbol{\xi}}-\widehat{\boldsymbol{\mu}})(\widetilde{\boldsymbol{\xi}}-\widehat{\boldsymbol{\mu}})^{T} d P(\widetilde{\boldsymbol{\xi}})=\widehat{\Sigma}
\end{gathered}
$$

We denote the dual variable of constraint (42b) by $q_{1}$, that of constraint (42c) by $\mathbf{h}$, and that of constraint (42d) by Q. The dual reformulation of problem (42) can be written as

$$
\begin{aligned}
& \max _{\left\{q_{1}, \mathbf{h}, \mathbf{Q}\right\}}-q_{1}-\mathbf{h}^{\mathrm{T}} \widehat{\mu}-\langle\mathbf{Q}, \widehat{\Sigma}\rangle, \\
& \text { s.t. } \widetilde{\xi}^{\mathrm{T}} \mathbf{B}_{1} \mathbf{w}+\widetilde{\xi}^{\mathrm{T}} \mathbf{B}_{2} \mathbf{w}+\widetilde{\xi}^{\mathrm{T}} \mathbf{H} \widetilde{\boldsymbol{\xi}}+q_{1}+\mathbf{h}^{\mathrm{T}} \widetilde{\xi} \\
&+(\widetilde{\boldsymbol{\xi}}-\widehat{\boldsymbol{\mu}})^{\mathrm{T}} \mathbf{Q}(\widetilde{\boldsymbol{\xi}}-\widehat{\boldsymbol{\mu}}) \geq 0, \quad \forall \widetilde{\xi} \in \mathbb{R}^{2 n} .
\end{aligned}
$$

Similarly, there is no dual gap between problems (42) and (43). Thus, the two problems have the same optimal objective function value. The equivalent matrix inequality of (43b) is (41b). Hence, we finish the proof of this theorem.

According to Theorems 3 and 4, we can obtain the equivalent SDP reformulation of model $R I M L^{-}$defined by (31), and the final formulation is as follows:

$$
\min _{\left\{p_{1}, \mathbf{v}, \mathbf{U}, q_{1}, \mathbf{h}, \mathbf{Q}\right\}} \lambda \cdot\left(p_{1}+\mathbf{v}^{T} \widehat{\mu}+\langle\mathbf{U}, \widehat{\Sigma}\rangle\right)+(1-\lambda) \cdot\left(q_{1}+\mathbf{h}^{T} \widehat{\mu}+\langle\mathbf{Q}, \widehat{\Sigma}\rangle\right),
$$

s.t. (31b), (34b), (34c), (41b).

\section{Empirical Experiments}

To investigate the performance of our model RIML, we conduct empirical experiments with comparison to models $\mathrm{RIML}^{-}$, SIML, and EW, where EW is the equally weighted model. We assume that an investor from the Chinese Mainland wants to invest RMB in four main international stock indexes, which are Nikkei 225 (Japan), Hang Seng Index (Hong Kong), S\&P 500 (USA), and FTSE 100 (UK). Market prices and spot exchange rates are from the database Wind (https://www.wind.com.cn/). We use weekly historical returns from March 26, 2004, to July 23, 2021 (883 observations). The in-sample period is set from March 26, 2004, to March 30, 2007, containing 150 historical return observations. The out-of-sample period is set from April 6, 2007, to July 23, 2021, containing 733 historical return observations. All experiments are conducted using MATLAB R2018a on an Intel Core i7 CPU 3.20 GHz desktop with $32 \mathrm{~GB}$ of RAM. We use MATLAB interface YALMIP by Lofberg [17]. SDP problems (RIML and $\mathrm{RIML}^{-}$) are solved by the Mosek package (https://www.mosek.com/), and linear programming problem (SIML) is solved by the Gurobi package (https://www.gurobi.com/).

Based on Delage and Ye [15], to compute the two parameters $\lambda_{1}$ and $\lambda_{2}$ of the ambiguity set of our model RIML in (9), we need to build uncertainty sets of the returns of assets and exchange rates. We first illustrate the samplebased mean returns, standard deviations, and covariance matrix of returns of assets and exchange rates during the insample period. Table 1 shows sample-based mean returns and standard deviations. From Table 1, we find that the standard deviations of returns of assets are much larger than those of exchange rates. Table 2 shows a sample-based covariance matrix. From Table 2, we find that the covariance of returns of assets is also much larger than those of exchange rates. Thus, we can conclude that the stock market is more volatile than the currency market. According to this observation, we assume that the size of the uncertainty set of returns of assets is larger than that of exchange rates. Specifically, we set the upper bound of returns of assets as 0.06 and the lower bound of those as -0.06 , whereas we set the upper bound of returns of exchange rates as 0.02 and the lower bound of those as -0.02 .

We conduct rolling forward experiments to assess the out-of-sample performances of models RIML, RIML ${ }^{-}$, SIML, and EW. We want to set the benchmark return $a$ as the realized mean return of model RIML in the in-sample period dynamically, and then $\operatorname{LPM}(\mathbf{w}, P)$ in (6) is similar to semivariance. However, if we do not know the benchmark return $a$ beforehand, we cannot compute the portfolio of model RIML; thus, we cannot acquire the realized mean return of model RIML in the in-sample period. Hence, instead, we use the realized mean return of model EW in the in-sample period to approximate that of model RIML in the in-sample period dynamically. Specifically, when the realized mean return of model EW is positive or zero, we set the benchmark return $a$ as three times of it. When the realized mean return of model EW is negative, we set $a$ as a third of it. Now we describe the procedure of our rolling forward 
TABle 1: Sample-based mean returns and standard deviations of assets and exchange rates during the in-sample period.

\begin{tabular}{lccccc}
\hline Asset & Mean & Standard deviation & Currency & Mean & Standard deviation \\
\hline N225 & 0.002887 & 0.022174 & JPYCNY & -0.001154 & 0.011226 \\
HSI & 0.003423 & 0.020297 & HKDCNY & -0.000474 & 0.001555 \\
SPX & 0.001875 & 0.014803 & USDCNY & -0.000458 & 0.001780 \\
FISE & 0.002670 & 0.014631 & GBPCNY & 0.000180 & 0.011438 \\
\hline
\end{tabular}

TABLE 2: Sample-based covariance matrix of returns of assets and exchange rates during the in-sample period.

\begin{tabular}{|c|c|c|c|c|c|c|c|c|}
\hline Covariance & N225 & HSI & SPX & FISE & JPYCNY & HKDCNY & USDCNY & GBPCNY \\
\hline N225 & $4.9167 e-4$ & & & & & & & \\
\hline HSI & $2.1037 e-4$ & $4.1199 e-4$ & & & & & & \\
\hline SPX & $1.6326 e-4$ & $1.3223 e-4$ & $2.1912 e-4$ & & & & & \\
\hline FISE & $1.8179 e-4$ & $1.2539 e-4$ & $1.5819 e-4$ & $2.1408 e-4$ & & & & \\
\hline JPYCNY & $-1.8237 e-5$ & $5.1719 e-5$ & $1.5598 e-6$ & $-3.4262 e-5$ & $1.2603 e-4$ & & & \\
\hline HKDCNY & $2.6835 e-7$ & $-3.6671 e-6$ & $6.0277 e-8$ & $2.2379 e-7$ & $2.1178 e-6$ & $2.4194 e-6$ & & \\
\hline USDCNY & $-6.6258 e-8$ & $-5.0596 e-6$ & $-1.4664 e-6$ & $1.5952 e-7$ & $3.3600 e-7$ & $2.4910 e-6$ & $3.1682 e-6$ & \\
\hline GBPCNY & $-4.2339 e-6$ & $7.0252 e-5$ & $1.7282 e-5$ & $-3.3184 e-5$ & $6.9690 e-5$ & $1.5398 e-6$ & $8.5962 e-7$ & $1.3084 e-4$ \\
\hline
\end{tabular}

experiment as follows. First, we use the 150 return observations in the in-sample period from March 26, 2004, to March 30, 2007, to compute the relevant parameters of models RIML and RIML ${ }^{-}$, and determine the three optimal portfolios of models RIML, RIML ${ }^{-}$, and SIML. According to the realized returns of assets and exchange rates in the first week during the out-of-sample period, we can compute the realized returns of the above three optimal portfolios and the equally weighted strategy. Then, we move the in-sample period one week forward by adding the new week and delete the first week. Based on the return observations in the new in-sample period, we can also compute the three new optimal portfolios of models RIML, RIML ${ }^{-}$, and SIML. Using the returns observations in the second week of the out-ofsample period and the optimal portfolios obtained from the in-sample period, we can also derive the realized returns of the four models. We continue this procedure until July 23, 2021. Consequently, we obtain four return series with 733 realized returns of the four models. Based on these four return series, we assess the realized performances of the four models using various performance measures, which are mean return, Sharpe ratio [18], downside Sharpe ratio [19], upside potential and downside risk (UP) ratio [20], Mean/ $\mathrm{VaR}$, Mean/CVaR, and the cumulative return at July 23, 2021, where VaR and CVaR are at the 0.95 level, Mean/VaR denotes Mean divided by $\mathrm{VaR}$, and Mean/CVaR denotes Mean divided by $\mathrm{CVaR}$. Mean return and the cumulative return on July 23, 2021, are return measures. Sharpe ratio, downside Sharpe ratio, UP ratio, Mean/VaR, and Mean/ $\mathrm{CVaR}$ are risk-adjusted return measures. According to Sortino and Meer [20], the UP ratio can be defined as follows:

$$
U P=\frac{1 / K \sum_{t=1}^{K} \max \left[0, r_{t}-\rho_{t}\right]}{\sqrt{1 / K \sum_{t=1}^{K}\left(\max \left[0, \rho_{t}-r_{t}\right]\right)^{2}}}
$$

where $r_{t}$ is the realized return of a portfolio at the $t$-th period and $\rho_{t}$ is a benchmark return at the same period, $t=1,2, \ldots, K$. Obviously, the UP ratio is an appropriate measure to assess the performance of portfolios with asymmetry distributions. Without loss of generality, in our numerical experiments, we set that $\rho_{t}=0, t=1,2, \ldots, K$.

For a portfolio $\mathbf{w}, W L P M\left(\mathbf{w}, \mathscr{P}_{1}\right)$ in $(10)$ is much larger than $W \operatorname{Return}\left(\mathbf{w}, \mathscr{P}_{1}\right)$ in (11). Hence, in order to balance $\operatorname{WLPM}\left(\mathbf{w}, \mathscr{P}_{1}\right)$ and $W \operatorname{Return}\left(\mathbf{w}, \mathscr{P}_{1}\right)$ and acquire a portfolio that has a good performance in terms of risk-adjusted return, we should set the risk aversion coefficient $\lambda$ small. In our empirical experiments, we consider various cases of $\lambda$, which are $\lambda=0.03, \lambda=0.02, \lambda=0.01, \lambda=0.009, \lambda=0.008$, $\lambda=0.007, \quad \lambda=0.006, \quad \lambda=0.005, \quad \lambda=0.004, \quad \lambda=0.003$, $\lambda=0.002$, and $\lambda=0.001$. Table 3 shows the realized performances of models RIML, RIML ${ }^{-}$, SIML, and EW in terms of the above various performance measures when $\lambda=0.03$. Table 4 shows the result when $\lambda=0.02$. Table 5 shows the result when $\lambda=0.01$. Table 6 shows the result when $\lambda=0.009$. Table 7 shows the result when $\lambda=0.008$. Table 8 shows the result when $\lambda=0.007$. Table 9 shows the result when $\lambda=0.006$. Table 10 shows the result when $\lambda=0.005$. Table 11 shows the result when $\lambda=0.004$. Table 12 shows the result when $\lambda=0.003$. Table 13 shows the result when $\lambda=0.002$. Table 14 shows the result when $\lambda=0.001$. In order to test whether the Sharpe ratio of our model RIML outperforms those of other models significantly, we employ a significance testing method about Sharpe ratios proposed by Jobson and Korkie [21]. The corresponding one-sided $p$ values are presented in the column of the Sharpe ratio. $* * *, * *$, and $*$ indicate that the Sharpe ratio of our model RIML outperforms that of the corresponding model significantly at the $1 \%$ level, $5 \%$ level, and $10 \%$ level, respectively. For all cases of the risk aversion coefficient $\lambda$, our model RIML consistently performs best in terms of return and risk-adjusted return measures among the four models. The Sharpe ratio of our model RIML is significantly larger than those of the other three models. Interestingly, we find that the realized performances of models RIML $^{-}$and SIML are very similar. For all performance measures, our model 
TABLE 3: Realized performances of models RIML, RIML ${ }^{-}$, SIML, and EW according to various performance measures when $\lambda=0.03$.

\begin{tabular}{lccccccc}
\hline & Mean & Sharpe ratio & Downside Sharpe & UP ratio & Mean/VaR & Mean/CVaR & Cumulative return \\
\hline RIML & 0.001705 & 0.059488 & 0.058076 & 0.514327 & 0.037829 & 0.001223 & 3.351051 \\
RIML $^{-}$ & 0.001370 & $0.047292^{* *}(0.022363)$ & 0.046309 & 0.509334 & 0.029937 & 0.000975 & 2.504079 \\
SIML & 0.001348 & $0.046351^{* *}(0.016264)$ & 0.045297 & 0.506573 & 0.029458 & 0.000949 & 2.359805 \\
EW & 0.000742 & $0.029613^{* *}(0.049575)$ & 0.027876 & 0.470055 & 0.019598 & 0.000605 & 1.826263 \\
\hline
\end{tabular}

TABLE 4: Realized performances of models RIML, RIML ${ }^{-}$, SIML, and EW according to various performance measures when $\lambda=0.02$.

\begin{tabular}{lccccccc}
\hline & Mean & Sharpe ratio & Downside Sharpe & UP ratio & Mean/VaR & Mean/CVaR & Cumulative return \\
\hline RIML & 0.001726 & 0.060390 & 0.059258 & 0.519263 & 0.038103 & 0.001242 & 3.475284 \\
RIML $^{-}$ & 0.001295 & $0.044441^{* * *}(0.004550)$ & 0.043359 & 0.504967 & 0.028298 & 0.000911 & 2.282259 \\
SIML & 0.001290 & $0.044243^{* * *}(0.004131)$ & 0.043196 & 0.504939 & 0.028195 & 0.000907 & 2.286590 \\
EW & 0.000742 & $0.029613^{* *}(0.046820)$ & 0.027876 & 0.470055 & 0.019598 & 0.000605 & 1.826263 \\
\hline
\end{tabular}

TABLE 5: Realized performances of models RIML, RIML ${ }^{-}$, SIML, and EW according to various performance measures when $\lambda=0.01$.

\begin{tabular}{lccccccc}
\hline & Mean & Sharpe ratio & Downside Sharpe & UP ratio & Mean/VaR & Mean/CVaR & Cumulative return \\
\hline RIML $^{-0.001703}$ & 0.059507 & 0.058433 & 0.518954 & 0.037254 & 0.001223 & 3.565365 \\
RIML $^{-}$ & 0.001283 & $0.043964^{* * *}(0.004513)$ & 0.042915 & 0.505353 & 0.028044 & 0.000902 & 2.265623 \\
SIML & 0.001286 & $0.044053^{* * *}(0.004818)$ & 0.043006 & 0.505152 & 0.028105 & 0.000903 & 2.245739 \\
EW & 0.000742 & $0.029613^{*}(0.052438)$ & 0.027876 & 0.470055 & 0.019598 & 0.000605 & 1.826263 \\
\hline
\end{tabular}

TABLE 6: Realized performances of models RIML, RIML ${ }^{-}$, SIML, and EW according to various performance measures when $\lambda=0.009$.

\begin{tabular}{lccccccc}
\hline & Mean & Sharpe ratio & Downside Sharpe & UP ratio & Mean/VaR & Mean/CVaR & Cumulative return \\
\hline RIML $^{-}$ & 0.001699 & 0.059338 & 0.058265 & 0.518775 & 0.037128 & 0.001220 & 3.597911 \\
RIML $^{-}$ & 0.001283 & $0.043950^{* * *}(0.004719)$ & 0.042902 & 0.505258 & 0.028037 & 0.000902 & 2.256801 \\
SIML & 0.001280 & $0.043853^{* * *}(0.004732)$ & 0.042812 & 0.504836 & 0.027976 & 0.000899 & 2.224514 \\
EW & 0.000742 & $0.029613^{*}(0.053487)$ & 0.027876 & 0.470055 & 0.019598 & 0.000605 & 1.826263 \\
\hline
\end{tabular}

TABLE 7: Realized performances of models RIML, RIML ${ }^{-}$, SIML, and EW according to various performance measures when $\lambda=0.008$.

\begin{tabular}{lccccccc}
\hline & Mean & Sharpe ratio & Downside Sharpe & UP ratio & Mean/VaR & Mean/CVaR & Cumulative return \\
\hline RIML $^{-0.001693}$ & 0.059139 & 0.058068 & 0.518561 & 0.037014 & 0.001216 & 3.623607 \\
RIML $^{-}$ & 0.001285 & $0.044023^{* * *}(0.005261)$ & 0.042975 & 0.505304 & 0.028084 & 0.000903 & 2.247054 \\
SIML & 0.001271 & $0.043547^{* * *}(0.004598)$ & 0.042515 & 0.504746 & 0.027783 & 0.000893 & 2.207364 \\
EW & 0.000742 & $0.029613^{*}(0.054733)$ & 0.027876 & 0.470055 & 0.019598 & 0.000605 & 1.826263 \\
\hline
\end{tabular}

TABLE 8: Realized performances of models RIML, RIML ${ }^{-}$, SIML, and EW according to various performance measures when $\lambda=0.007$.

\begin{tabular}{lccccccc}
\hline & Mean & Sharpe ratio & Downside Sharpe & UP ratio & Mean/VaR & Mean/CVaR & Cumulative return \\
\hline RIML $^{-}$ & 0.001688 & 0.058926 & 0.057856 & 0.518395 & 0.036891 & 0.001211 & 3.620472 \\
RIML $^{-}$ & 0.001290 & $0.044194^{* * *}(0.006244)$ & 0.043147 & 0.505530 & 0.028196 & 0.000907 & 2.246750 \\
SIML & 0.001281 & $0.043870^{* * *}(0.005724)$ & 0.042836 & 0.505163 & 0.027993 & 0.000900 & 2.222131 \\
EW & 0.000742 & $0.029613^{*}(0.056102)$ & 0.027876 & 0.470055 & 0.019598 & 0.000605 & 1.826263 \\
\hline
\end{tabular}

TABLE 9: Realized performances of models RIML, RIML ${ }^{-}$, SIML, and EW according to various performance measures when $\lambda=0.006$.

\begin{tabular}{lccccccc}
\hline & Mean & Sharpe ratio & Downside Sharpe & UP ratio & Mean/VaR & Mean/CVaR & Cumulative return \\
\hline RIML $^{-}$ & 0.001683 & 0.058726 & 0.057660 & 0.518251 & 0.036779 & 0.001207 & 3.611440 \\
RIML $^{-}$ & 0.001290 & $0.044200^{* * *}(0.006894)$ & 0.043154 & 0.505624 & 0.028203 & 0.000907 & 2.236750 \\
SIML $^{0.001287}$ & $0.044086^{* * *}(0.006774)$ & 0.043048 & 0.505264 & 0.028130 & 0.000904 & 2.235418 \\
EW & 0.000742 & $0.029613^{*}(0.057409)$ & 0.027876 & 0.470055 & 0.019598 & 0.000605 & 1.826263 \\
\hline
\end{tabular}


TABLE 10: Realized performances of models RIML, RIML ${ }^{-}$, SIML, and EW according to various performance measures when $\lambda=0.005$.

\begin{tabular}{lccccccc}
\hline & Mean & Sharpe ratio & Downside Sharpe & UP ratio & Mean/VaR & Mean/CVaR & Cumulative return \\
\hline RIML & 0.001678 & 0.058527 & 0.057466 & 0.505743 & 0.036670 & 0.001203 & 3.597434 \\
RIML $^{-}$ & 0.001291 & $0.044209^{* * *}(0.007266)$ & 0.043167 & 0.505743 & 0.028213 & 0.000907 & 2.236180 \\
SIML & 0.001295 & $0.044358^{* * *}(0.007821)$ & 0.043314 & 0.505397 & 0.028301 & 0.000910 & 2.249519 \\
EW & 0.000742 & $0.029613^{*}(0.058741)$ & 0.027876 & 0.470055 & 0.019598 & 0.000605 & 1.826263 \\
\hline
\end{tabular}

TABLE 11: Realized performances of models RIML, RIML ${ }^{-}$, SIML, and EW according to various performance measures when $\lambda=0.004$.

\begin{tabular}{lccccccc}
\hline & Mean & Sharpe ratio & Downside Sharpe & UP ratio & Mean/VaR & Mean/CVaR & Cumulative return \\
\hline RIML $^{-}$ & 0.001672 & 0.058311 & 0.057255 & 0.517977 & 0.036551 & 0.001198 & 3.582173 \\
RIML $^{-}$ & 0.001298 & $0.044450^{* * *}(0.008215)$ & 0.043414 & 0.506065 & 0.028373 & 0.000912 & 2.245132 \\
SIML & 0.001318 & $0.045132^{* *}(0.010416)$ & 0.044098 & 0.506448 & 0.028813 & 0.000926 & 2.281277 \\
EW & 0.000742 & $0.029613^{*}(0.060217)$ & 0.027876 & 0.470055 & 0.019598 & 0.000605 & 1.826263 \\
\hline
\end{tabular}

TABLE 12: Realized performances of models RIML, RIML ${ }^{-}$, SIML, and EW according to various performance measures when $\lambda=0.003$.

\begin{tabular}{lccccccc}
\hline & Mean & Sharpe ratio & Downside Sharpe & UP ratio & Mean/VaR & Mean/CVaR & Cumulative return \\
\hline RIML $^{-}$ & 0.001667 & 0.058083 & 0.057032 & 0.517816 & 0.036426 & 0.001194 & 3.552670 \\
RIML $^{-}$ & 0.001318 & $0.045117^{* *}(0.011217)$ & 0.044097 & 0.506885 & 0.028816 & 0.000927 & 2.273388 \\
SIML & 0.001344 & $0.045975^{* *}(0.015369)$ & 0.044981 & 0.507586 & 0.029386 & 0.000945 & 2.307677 \\
EW & 0.000742 & $0.029613^{*}(0.061814)$ & 0.027876 & 0.470055 & 0.019598 & 0.000605 & 1.826263 \\
\hline
\end{tabular}

TABLE 13: Realized performances of models RIML, $\mathrm{RIML}^{-}$, SIML, and EW according to various performance measures when $\lambda=0.002$.

\begin{tabular}{lccccccc}
\hline & Mean & Sharpe ratio & Downside Sharpe & UP ratio & Mean/VaR & Mean/CVaR & Cumulative return \\
\hline RIML $^{-0.001661}$ & 0.057843 & 0.056797 & 0.517629 & 0.036296 & 0.001189 & 3.556490 \\
RIML $^{-}$ & 0.001337 & $0.045719^{* *}(0.016084)$ & 0.044725 & 0.507588 & 0.029223 & 0.000940 & 2.293018 \\
SIML & 0.001335 & $0.045646^{* *}(0.016097)$ & 0.044649 & 0.507032 & 0.029169 & 0.000938 & 2.287590 \\
EW & 0.000742 & $0.029613^{*}(0.063534)$ & 0.027876 & 0.470055 & 0.019598 & 0.000605 & 1.826263 \\
\hline
\end{tabular}

TABLE 14: Realized performances of models RIML, RIML ${ }^{-}$, SIML, and EW according to various performance measures when $\lambda=0.001$.

\begin{tabular}{lccccccc}
\hline & Mean & Sharpe ratio & Downside Sharpe & UP ratio & Mean/VaR & Mean/CVaR & Cumulative return \\
\hline RIML $^{-}$ & 0.001654 & 0.057576 & 0.056534 & 0.517410 & 0.036149 & 0.001183 & 3.542119 \\
RIML $^{-}$ & 0.001341 & $0.045846^{* *}(0.020987)$ & 0.044856 & 0.507387 & 0.029304 & 0.000942 & 2.287160 \\
SIML & 0.001351 & $0.046203^{* *}(0.024616)$ & 0.045205 & 0.507646 & 0.029533 & 0.000949 & 2.288830 \\
EW & 0.000742 & $0.029613^{*}(0.065501)$ & 0.027876 & 0.470055 & 0.019598 & 0.000605 & 1.826263 \\
\hline
\end{tabular}

RIML outperforms models RIML $^{-}$and SIML. The experimental result demonstrates the benefit of accounting for moments ambiguity in a robust international portfolio optimization model using first-order LPM as the risk measure. For all cases of the risk aversion coefficient $\lambda$, the model EW consistently has the worst performance in terms of return and risk-adjusted return measures. DeMiguel et al. [22] find that, in their numerical experiments, the equally weighted model EW outperforms sample-based mean-variance strategy and various extensions of sample-based meanvariance strategy, which are designed to deal with the problem of estimation error. Hence, the result that our model RIML significantly outperforms the model EW is very encouraging. Our empirical results show that when $\lambda$ is set small, the variation of $\lambda$ does not change the performances of models RIML, RIML ${ }^{-}$, and SIML significantly.

\section{Conclusions}

In this paper, we propose a robust international portfolio optimization model with worst-case LPM and mean return. In this model, we assume that the distributions and the firstand second-order moments of distributions of future returns of assets and exchange rates are ambiguous. We reformulate the proposed model into an equivalent SDP problem which is computationally tractable. For investigation of the performance of our proposed model, we also give two benchmark models. In the first benchmark model SIML, we use historical returns to form approximations of the distributions of future returns and build a scenario-based international portfolio optimization model under these approximations of distributions. In the second benchmark model $\mathrm{RIML}^{-}$, we assume that the distributions of future 
returns are ambiguous, but the first- and second-order moments of distributions are known beforehand. We also reformulate this model into an equivalent SDP problem. We conduct empirical experiments in a rolling forward way using the return measures and various risk-adjusted return measures to compare the out-of-sample performances of the four models RIML, RIML ${ }^{-}$, SIML, and an equally weighted model EW. The result demonstrates the superiority of our model RIML over other models. It shows that investors can get benefits when accounting for the ambiguity of the firstand second-order moments. It also verifies that robust models outperform scenario-based model and equally weighted model.

\section{Data Availability}

The data used to support the findings of this study are available from the database Wind with the web address https://www.wind.com.cn/.

\section{Conflicts of Interest}

The authors declare that they have no conflicts of interest.

\section{Acknowledgments}

This work was supported by the National Natural Science Foundation of China (nos. 71720107002 and 71971086), the Joint Foundation of National Natural Science Foundation of China-Guangdong Province (no. U1901223), the Foundation for Key Program of Ministry of Science and Technology of China (no. 2020AAA0108404), the Fundamental Research Funds for the Central Universities (nos. 2018JDXM02 and 2019ZD13), the Financial Service Innovation and Risk Management Research Base of Guangzhou of China, the Guangdong Basic and Applied Basic Research Foundation (no. 2019B151502037), and GDUPS (Liu Yongjun 2019).

\section{References}

[1] H. G. Grubel, "Internationally diversified portfolios: welfare gains and capital flows," The American Economic Review, vol. 58, pp. 1299-1314, 1968.

[2] H. Levy and M. Sarnat, "International diversification of investment portfolios," The American Economic Review, vol. 60, pp. 668-675, 1970.

[3] D. R. Lessard, "International portfolio diversification: a multivariate analysis for a group of Latin american countries," The Journal of Finance, vol. 28, no. 3, pp. 619-633, 1973.

[4] N. Coeurdacier and S. Guibaud, "International portfolio diversification is better than you think," Journal of International Money and Finance, vol. 30, no. 2, pp. 289-308, 2011.

[5] M. Umutlu and S. G. Yarg1, "To diversify or not to diversify internationally?" Finance Research Letters, vol. 44, Article ID 102110, 2022.

[6] M. Jasemi, L. Monplaisir, and P. Amini Jam, "Development of an efficient method to approximate the risk measure of lower partial moment of the first order," Computers \& Industrial Engineering, vol. 135, pp. 326-332, 2019.

[7] V. S. Bawa, "Optimal rules for ordering uncertain prospects," Journal of Financial Economics, vol. 2, no. 1, pp. 95-121, 1975.
[8] V. S. Bawa and E. B. Lindenberg, "Capital market equilibrium in a mean-lower partial moment framework," Journal of Financial Economics, vol. 5, no. 2, pp. 189-200, 1977.

[9] P. C. Fishburn, "Mean-risk analysis with risk associated with below-target returns," The American Economic Review, vol. 67, pp. 116-126, 1977.

[10] L. Chen, S. He, and S. Zhang, "Tight bounds for some risk measures, with applications to robust portfolio selection," Operations Research, vol. 59, no. 4, pp. 847-865, 2011.

[11] M. Ç. Pınar and A. B. Paç, "Mean semi-deviation from a target and robust portfolio choice under distribution and mean return ambiguity," Journal of Computational and Applied Mathematics, vol. 259, pp. 394-405, 2014.

[12] A. Ling, J. Sun, and X. Yang, "Robust tracking error portfolio selection with worst-case downside risk measures," Journal of Economic Dynamics and Control, vol. 39, pp. 178-207, 2014.

[13] A. Ling and L. Tang, "A numerical study for robust active portfolio management with worst-case downside risk measure," Mathematical Problems in Engineering, vol. 2014, Article ID 912389, 13 pages, 2014.

[14] A. Ling, J. Sun, and M. Wang, "Robust multi-period portfolio selection based on downside risk with asymmetrically distributed uncertainty set," European Journal of Operational Research, vol. 285, no. 1, pp. 81-95, 2020.

[15] E. Delage and Y. Ye, "Distributionally robust optimization under moment uncertainty with application to data-driven problems," Operations Research, vol. 58, no. 3, pp. 595-612, 2010.

[16] A. Shapiro, "On duality theory of conic linear problems," in Semi-infinite Programming, pp. 135-165, Springer, Berlin, Germany, 2001.

[17] J. Lofberg, "Yalmip: a toolbox for modeling and optimization in matlab," in Proceedings of the IEEE international conference on robotics and automation (IEEE Cat. No. 04CH37508), pp. 284-289, IEEE, Taipei, Taiwan, September 2004.

[18] W. F. Sharpe, “The sharpe ratio," Journal of Portfolio Management, vol. 21, no. 1, pp. 49-58, 1994.

[19] W. T. Ziemba, "The symmetric downside-risk sharpe ratio," Journal of Portfolio Management, vol. 32, no. 1, pp. 108-122, 2005.

[20] F. A. Sortino and R. V. D. Meer, "Downside risk," Journal of Portfolio Management, vol. 17, no. 4, pp. 27-31, 1991.

[21] J. D. Jobson and B. M. Korkie, "Performance hypothesis testing with the sharpe and treynor measures," The Journal of Finance, vol. 36, no. 4, pp. 889-908, 1981.

[22] V. DeMiguel, L. Garlappi, and R. Uppal, "Optimal versus naive diversification: how inefficient is the $1 /$ NPortfolio strategy?" Review of Financial Studies, vol. 22, no. 5, pp. 1915-1953, 2009. 